\title{
Correction to: Using Innovative Technologies as Therapeutic and Educational Tools for Children with Autism Spectrum Disorder
}

Eynat Gal, P. L. (Tamar) Weiss, and Massimo Zancanaro

\section{Correction to:}

Chapter 10 in: A. Rizzo, S. Bouchard (eds.), Virtual Reality for Psychological and Neurocognitive Interventions, Virtual Reality Technologies for Health and Clinical Applications, https://doi.org/10.1007/978-1-4939-9482-3_10

The surname of the co-author Massimo Zancanaro was misspelled as Zancanero in the previous version of this book. The same has been corrected in this revised version of this book. 